Bundesgesundheitsbl 2020 - 63:397-403 https://doi.org/10.1007/s00103-020-03105-y Online publiziert: 3 . März 2020

(c) Der/die Autor(en) 2020

\section{Einleitung}

Mit einer durchschnittlichen 12-MonatsPrävalenz von 7,1 \% bei Frauen und 5,4\% bei Männern ist Asthma bronchiale eine der häufigsten chronischen Erkrankungen in Deutschland [1], was es zu einer der wichtigsten Volkskrankheiten macht [2]. Asthma ist eine chronisch entzündliche Erkrankung der Atemwege, die durch eine bronchiale Überempfindlichkeit (Hyperreaktivität) und eine variable Atemwegsobstruktion charakterisiert ist [3].

Das im Kindes- oder Jugendalter auftretende Asthma bezeichnet man als Childhood- oder Early-onset Asthma (COA). In den meisten Fällen zeigen sich erste Symptome vor dem 6. Lebensjahr [4]. Man geht davon aus, dass Risikofaktoren für COA vor allem eine genetische Prädisposition direkt für Asthma sowie das Vorhandensein von allergischen Erkrankungen in der Familie sind. Weiterhin können virale respiratorische Infektionen, das Mikrobiom sowie eine frühkindliche Allergensensibilisie-

Weitere Informationen zu den Affiliations der Autoren befinden sich auf der letzten Artikelseite.

Susan Langer' · Johannes Horn' · Alexander Kluttig' • Rafael Mikolajczyk' Stefan Karrasch ${ }^{2,3,4}$. Holger Schulz ${ }^{2,4}$. Heinz-Erich Wichmann ${ }^{2}$. Jakob Linseisen ${ }^{5,6}$. Lina Jaeschke ${ }^{7}$. Tobias Pischon ${ }^{7,8,9,10}$ - Julia Fricke ${ }^{11}$ - Thomas Keil ${ }^{11,12,13}$. Wolfgang Ahrens ${ }^{14,15} \cdot$ Kathrin Günther $^{14}$. Oliver Kuß ${ }^{16} \cdot$ Tamara Schikowski $^{17}$.

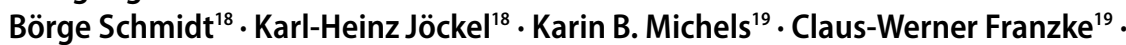
Heiko Becher ${ }^{20}$. Annika Jagodzinski ${ }^{21,22,23}$. Stefanie Castell ${ }^{24}$. Yvonne Kemmling ${ }^{24}$. Wolfgang Lieb ${ }^{25} \cdot$ Sabina Waniek ${ }^{25} \cdot$ Kerstin Wirkner $^{26,27} \cdot$ Markus Löffler $^{26,27}$. Rudolf Kaaks ${ }^{28} \cdot$ Karin Halina Greiser ${ }^{28}$. Klaus Berger ${ }^{29}$. Nicole Legath ${ }^{29}$. Claudia Meinke-Franze ${ }^{30}$. Sabine Schipf ${ }^{30}$. Michael Leitzmann ${ }^{31}$. Hansjörg Baurecht ${ }^{31} \cdot$ Korbinian Weig $^{32}$ - Efrat Amitay ${ }^{32}$. Cornelia Gottschick ${ }^{1}$

\title{
Häufigkeit von Asthma bronchiale und Alter bei der Erstdiagnose - erste Ergebnisse der NAKO Gesundheitsstudie
}

rung und auch Passivrauchen eine Rolle bei der Entstehung von Asthma in der Kindheit spielen [5].

Im Gegensatz zum vergleichsweise intensiv erforschten COA ist über das Lateoder Adult-onset Asthma (LOA/AOA) [6] noch relativ wenig bekannt. Ab welchem Alter man von Late- bzw. Adultonset Asthma spricht, ist nicht eindeutig definiert $[6,7]$ und je nach Definition kann dies bereits ab dem 12. oder erst $\mathrm{ab}$ dem 65. Lebensjahr beginnen [8, 9]. Adult-onset Asthma ist seltener als COA mit Allergien assoziiert, es geht häufiger mit Übergewicht einher als COA und wird häufiger bei Frauen beobachtet als bei Männern [10]. Ebenso können bei der Entstehung des LOA bzw. AOA berufliche Expositionen eine Rolle spielen [7]. Es ist zudem durch eine schlechtere Lungenfunktion $[10,11]$ und eine niedrigere Remissionsrate als COA charakterisiert $[10,12]$. Eine US-amerikanische Studie zeigte eine 5-Jahres-Inzidenz bei über 65-Jährigen von 103/100.000 Personenjahre [13]. In der Studie zur Gesundheit Erwachsener (DEGS) 1 wurde nicht zwischen beiden Formen des Asthmas getrennt und altersstratifizierte Prävalenzen unabhängig vom Zeitpunkt der Erstdiagnose berichtet. Es gibt in Deutsch- land bisher keine Forschungsarbeit, die sich explizit mit AOA befasst [14].

Unsere aktuelle Arbeit beschreibt, inwiefern die in der NAKO Gesundheitsstudie erhobenen Daten zur Halbzeit der Basiserhebung die aus anderen Quellen bekannten Daten zu Asthma widerspiegeln. Zudem wird das berichtete Alter bei Erstdiagnose der NAKO-Studienteilnehmenden analysiert, um so den Anteil derjenigen $\mathrm{zu}$ ermitteln, die von COA bzw. AOA betroffen sind.

\section{Material und Methoden}

Die vorliegende Analyse beruht auf einem Datensatz zur Halbzeit der Basiserhebung der NAKO mit 101.723 Teilnehmerinnen und Teilnehmern, die von März 2014 bis März 2017 in insgesamt 18 Studienzentren untersucht wurden. Die NAKO ist eine bundesweite bevölkerungsbezogene Kohortenstudie mit über 200.000 Teilnehmenden. Zum gegenwärtigen Zeitpunkt stehen bereinigte Daten der 18 Studienzentren von 101.723 Personen zur Verfügung, welche eine erste Auswertung ermöglichen. Das Altersspektrum der teilnehmenden Personen lag bei der Rekrutierung bei 20-69 Jahren, da aber von der Stich- 


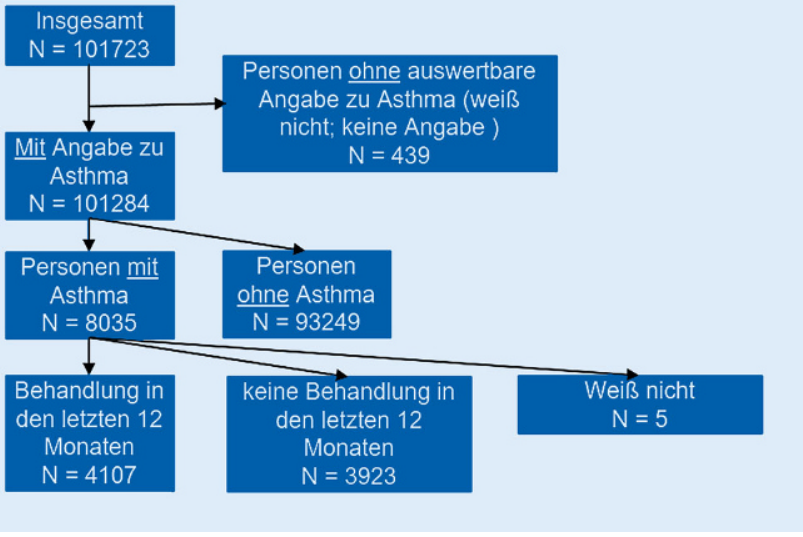

Abb. $1<$ Studienpopulation zur Halbzeit der NAKOBasiserhebung bzgl. der Antworten auf Fragen zu Asthma probenziehung bis zur Untersuchung teilweise 5 Jahre vergingen, sind Daten von Personen bis $\mathrm{zu}$ einem Alter von 75 Jahren (>69 Jahre: $N=2962$ ) enthalten. Da in den DEGS-Daten die oberste Altersgruppe 70-79 Jahre ist, kann nur diese hier zum Vergleich herangezogen werden. Detaillierte Beschreibungen zum Studiendesign und zur Rekrutierung der Teilnehmenden sind den Designpublikationen zu entnehmen [15, 16].

In die hier vorliegende Analyse gingen die Variablen Alter, Geschlecht sowie die Antworten zu folgenden Fragen aus dem persönlichen Interview ein: 1) „Wurde bei ihnen jemals Asthma bronchiale von einem Arzt diagnostiziert?“, 2) „Wenn ja, in welchem Alter/Jahr zum ersten Mal?“ und 3) „Wurden Sie in den letzten $12 \mathrm{Mo-}$ naten wegen Asthma bronchiale ärztlich behandelt?"

Die NAKO-Daten wurden mit den Daten der Studie zur Gesundheit Erwachsener in Deutschland DEGS (1; [17]; 2008-2011) mit 7896 Personen verglichen. Bei dieser Studie handelt es sich um einen Teil des Gesundheitsmonitorings des Robert Koch-Instituts (RKI), in dem seit 2008 bundesweit repräsentative Gesundheitsdaten bei Personen mit einem Altersspektrum von 18-79 Jahren erhoben werden. Die DEGS-(1)-Daten wurden den Public-Use-Files entnommen und beinhalteten die Variablen Alter, Geschlecht sowie die Antworten auf die Fragen, ob jemals Asthma bronchiale vom Arzt diagnostiziert wurde und ob Asthma in den letzten 3 Monaten behandelt wurde oder eine ärztliche Behand- lung in den folgenden 3 Monaten geplant ist.

Erstdiagnosen von Asthma vor dem 18. Geburtstag wurden in dieser Analyse als Childhood-onset Asthma (COA) sowie Erstdiagnosen im Erwachsenenalter als Adult-onset Asthma (AOA) definiert. Die Angaben zur Häufigkeit von Asthma wurden stratifiziert nach Alter und Geschlecht dargestellt und mit 95\%Konfidenzintervallen (Clopper-Pearson) ergänzt. Statistische Auswertungen wurden mit R 3.6.0 durchgeführt.

\section{Ergebnisse}

Von 101.723 Teilnehmenden wurden 439 Beobachtungen wegen fehlender Angaben ausgeschlossen, sodass insgesamt 101.284 Personen in die folgenden Analysen eingeschlossen wurden. Insgesamt gaben 8035 (7,9\%; $95 \%$ KI [7,8\%, $8,1 \%]$ ) Personen an, dass bei ihnen jemals im Laufe ihres Lebens Asthma von einem Arzt diagnostiziert wurde (• Abb. 1). Von diesen gaben 51,1\% $(N=4107)$ an, in den letzten $12 \mathrm{Mo-}$ naten behandelt worden $\mathrm{zu}$ sein $(N=5$ ohne Angabe). Frauen waren mit 8,7\% $(N=4747 ; 95 \%$ KI $[8,5 \%, 9,0 \%])$ häufiger betroffen als Männer mit 7,0\% $(N=3288 ; 95 \%$ KI [6,8\%, 7,2\%]). In der DEGS-Studie $(N=7896)$ waren die Zahlen für jemals diagnostiziertes Asthma mit $9,5 \%$ bei Frauen $(N=393 ; 95 \% \mathrm{KI}$ $[8,6 \%, 10,4 \%])$ und $6,8 \%$ bei Männern $(N=257 ; 95 \%$ KI $[6,1 \%, 7,7 \%])$ ähnlich. In beiden Studien waren insbesondere in den höheren Altersklassen Frauen häufiger von Asthma betroffen als Männer (• Abb. 2). Noch deutlicher wird dieser
Geschlechterunterschied, wenn man nur die Teilnehmenden mit Asthma betrachtet, die momentan in Behandlung sind. In der NAKO waren $4,7 \%$ der Frauen $(N=2561 ; 95 \%$ KI $[4,5 \%, 4,8 \%])$, aber nur 3,3\% der Männer $(N=1546$; $95 \% \mathrm{KI}[3,1 \%, 3,5 \%])$ in den letzten 12 Monaten wegen Asthma in ärztlicher Behandlung. Das entspricht 53,9\% der Frauen (95\% KI [52,5\%, 55,3\%]) bzw. $47,0 \%$ der Männer (95\% KI [45,3\%, $48,7 \%]$ ), bei denen jemals Asthma diagnostiziert wurde. In DEGS waren 4,0\% der Frauen, aber nur 1,7\% der Männer in ärztlicher Behandlung (3 Monate davor oder geplant in den nächsten 3 Monaten), was einem Anteil von 38,2\% bzw. 23,7\% aller, bei denen jemals Asthma diagnostiziert wurde, entspricht.

In beiden Studien und jeweils bei beiden Geschlechtern waren die jüngeren Altersstrata häufiger (jemals) von Asthma betroffen als die höheren Altersstrata. Am häufigsten war Asthma in der jüngsten Alterskategorie bei den Frauen vertreten: in der NAKO (20-29 Jahre) mit 10,3\% (95\% KI [9,4\%; 11,2\%]) sowie in DEGS (18-29 Jahre) mit 13,5\% (95\% KI [10,7\%; 16,7\%]). In den gleichen Altersstrata hatten in der NAKO 10,0\% der Männer Asthma (95\% KI [8,9\%; $11,1 \%]$ ), in DEGS waren es $10,8 \%$ (95\% KI $[8,2 \% ; 13,8 \%])$. Im Gegensatz da$\mathrm{zu}$ waren in den höchsten Altersstrata (70-75 Jahre NAKO; 70-79 Jahre DEGS) $8,7 \%$ der Frauen in der NAKO von Asthma betroffen (95\% KI [7,3\%; 10,3\%]) und $8,0 \%$ der Frauen in DEGS (95\% KI $[6,0 \% ; 10,4 \%])$ sowie 5,8\% der Männer in der NAKO (95\% KI [4,7\%; 7,1\%]) bzw. 4,6\% der Männer in DEGS (95\% KI $[3,0 \% ; 6,6 \%])$.

Eine Stratifizierung der NAKO-Teilnehmenden nach Geburtsjahr zeigt, dass $10,4 \%$ der jüngsten Geburtsjahrgänge (1990-1999) in ihrem bisherigen Leben bereits eine Asthmadiagnose erhielten (- Abb. 3). Im Vergleich dazu lag diese Zahl in den ältesten Geburtsjahrgängen der NAKO (1940-1949) bei nur 7,4\% (Frauen 10\%, Männer 6\%) für das gesamte bisherige Leben. Der Anstieg der kumulativen Asthmadiagnosen ist damit bei den jüngeren Geburtsjahrgängen deutlich steiler. 
Bundesgesundheitsbl 2020 ·63:397-403 https://doi.org/10.1007/s00103-020-03105-y

(c) Der/die Autor(en) 2020

S. Langer · J. Horn · A. Kluttig · R. Mikolajczyk · S. Karrasch · H. Schulz · H.-E. Wichmann · J. Linseisen · L. Jaeschke · T. Pischon · J. Fricke · T. Keil · W. Ahrens · K. Günther · O. Kuß · T. Schikowski · B. Schmidt · K.-H. Jöckel · K. B. Michels · C.-W. Franzke · H. Becher · A. Jagodzinski · S. Castell · Y. Kemmling · W. Lieb · S. Waniek · K. Wirkner · M. Löffler · R. Kaaks · K. H. Greiser · K. Berger · N. Legath · C. Meinke-Franze · S. Schipf · M. Leitzmann $\cdot$ H. Baurecht $\cdot$ K. Weigl $\cdot$ E. Amitay $\cdot$ C. Gottschick

\section{Häufigkeit von Asthma bronchiale und Alter bei der Erstdiagnose - erste Ergebnisse der NAKO Gesundheitsstudie}

\section{Zusammenfassung}

Hintergrund. Asthma bronchiale stellt über alle Bildungsschichten hinweg eine der häufigsten chronischen Erkrankungen im Kindes- und Erwachsenenalter dar. Im Erwachsenenalter erstmals auftretendes Asthma (Adult-onset Asthma, AOA) ist im Vergleich zu Childhood-onset Asthma (COA) mit einer schlechteren Prognose assoziiert, was verstärkte Untersuchungen in diesem Bereich erfordert. Ziel der Arbeit war es, Häufigkeiten und das Alter bei Erstdiagnose aus Selbstangaben zu Asthma bei Erwachsenen in der NAKO Gesundheitsstudie darzustellen und mit Daten der Studie zur Gesundheit Erwachsener in Deutschland (DEGS) zu vergleichen.
Material und Methoden. Unsere Analyse beruht auf einem Datensatz zur Halbzeit der NAKO-Basiserhebung mit 101.284 Personen. Verwendete Variablen waren die Selbstangabe zum jemals ärztlich diagnostizierten Asthma, Alter bei Erstdiagnose, Behandlung in den letzten 12 Monaten, Alter und Geschlecht. Ergebnisse. Laut Datenbasis zur Halbzeit berichteten in der NAKO 8,7\% der Frauen und 7,0\% der Männer, dass bei innen jemals Asthma diagnostiziert wurde.

Etwa bei einem Drittel der Teilnehmenden mit Asthma wurde die Diagnose bereits im Kindes-/Jugendalter ( $<18$ Jahre) gestellt. Dabei waren 2,2\% der Frauen und 2,8\% der Männer von COA betroffen und 6,5\% der Frauen und 4,2\% der Männer von AOA. In den vorangegangenen 12 Monaten gaben $33 \%$ der COA-Fälle und $60 \%$ der AOA-Fälle an, ärztlich behandelt worden zu sein.

Diskussion. Die Häufigkeit von Asthma in der NAKO Gesundheitsstudie in Abhängigkeit von Alter und Geschlecht entspricht weitgehend den Daten aus anderen Studien wie DEGS. Allerdings ist der AOA-Anteil höher als in der Literatur beschrieben. Der Anstieg der kumulativen Asthmadiagnosen ist bei den jüngeren Geburtsjahrgängen deutlich steiler, was für eine Zunahme über die Zeit spricht.

Schlüsselwörter

Asthma - Respiratorische Erkrankung · Chronische Erkrankungen · Allgemeinbevölkerung · Kohortenstudie

\section{Occurrence of bronchial asthma and age at initial asthma diagnosis_first results of the German National Cohort}

\section{Abstract}

Background. Asthma is one of the most common chronic diseases in both children and adults. Asthma first occurring in adulthood (adult-onset asthma, AOA) is associated with poorer prognosis compared to childhoodonset asthma (COA), which urgently calls for more research in this area. The aim of this work was to analyze the data on asthma collected in the German National Cohort and compare it with the German Health Interview and Examination Survey for Adults (DEGS), in particular regarding AOA.

Material and Methods. Our analysis was based on the dataset of the main questionnaire at mid-term of the German National Cohort baseline examination, comprising
101,723 participants. Variables considered in the analyses were self-reported diagnosis of asthma, age at first diagnosis, asthma treatment in the past 12 months, age, and sex. Results. In the midterm dataset, $8.7 \%$ of women and $7.0 \%$ of men in the German National Cohort reported that they had ever been diagnosed with asthma. Approximately one third of participants with asthma received their initial diagnosis before their 18th birthday. COA affected $2.2 \%$ of women and $2.8 \%$ of men, whereas AOA affected $6.5 \%$ of women and $4.2 \%$ of men. During the previous 12 months, $33 \%$ of COA cases and $60 \%$ of AOA cases were medically treated.
Conclusion. The proportion of persons affected by asthma in the German National Cohort, as well as observed patterns regarding age and gender, corresponds to other data sources such as DEGS. However, in our analysis, the proportion of individuals with AOA was higher than described in the literature. The increase in cumulative asthma diagnoses with age is markedly steeper in younger participants, indicating a rising trend over time.

\section{Keywords}

Asthma - Chronic diseases - Respiratory diseases - General population - Cohort study
Nach der Definition für AOA ab 18 Jahren waren $31 \%(N=2512)$ der asthmaerkrankten NAKO-Teilnehmenden von COA betroffen und $67 \%$ von AOA $(N=5345 ; N=178$ ohne Altersangabe für Erstdiagnose). Während von allen COA-Betroffenen 51,4\% männlich und $48,6 \%$ weiblich waren, verschob sich dieses Verhältnis bei AOA zu den Frauen auf 63,8 \% (Männer 36,2\%). Von allen COA-Betroffenen gaben nur 37\% der Frauen (95\% KI [34,7\%, 40,2\%]) und $30 \%$ der Männer (95\% KI [27,3\%, $32,3 \%]$ ) an, in den letzten 12 Monaten ärztlich wegen Asthma behandelt worden zu sein. Bei AOA waren dies $60 \%$ der Frauen (95\% KI [58,0\%, 61,2\%]) und $58 \%$ der Männer (95\% KI [56,0\%, $60,3 \%])$.
Von allen teilnehmenden Frauen waren $2,2 \%$ von COA betroffen $(N=1221$; $95 \% \mathrm{KI}[2,1 \%, 2,4 \%])$ und $6,5 \%$ von AOA $(N=3526 ; 95 \%$ KI $[6,3 \%, 6,7 \%])$. Im Gegensatz dazu waren bei den Männern $2,7 \%$ von COA $(N=1291 ; 95 \%$ KI $[2,6 \%, 2,9 \%])$ und $4,2 \%$ von $\mathrm{AOA}$ $(N=1997 ; 95 \%$ KI $[4,1 \%, 4,4 \%])$ betroffen. 


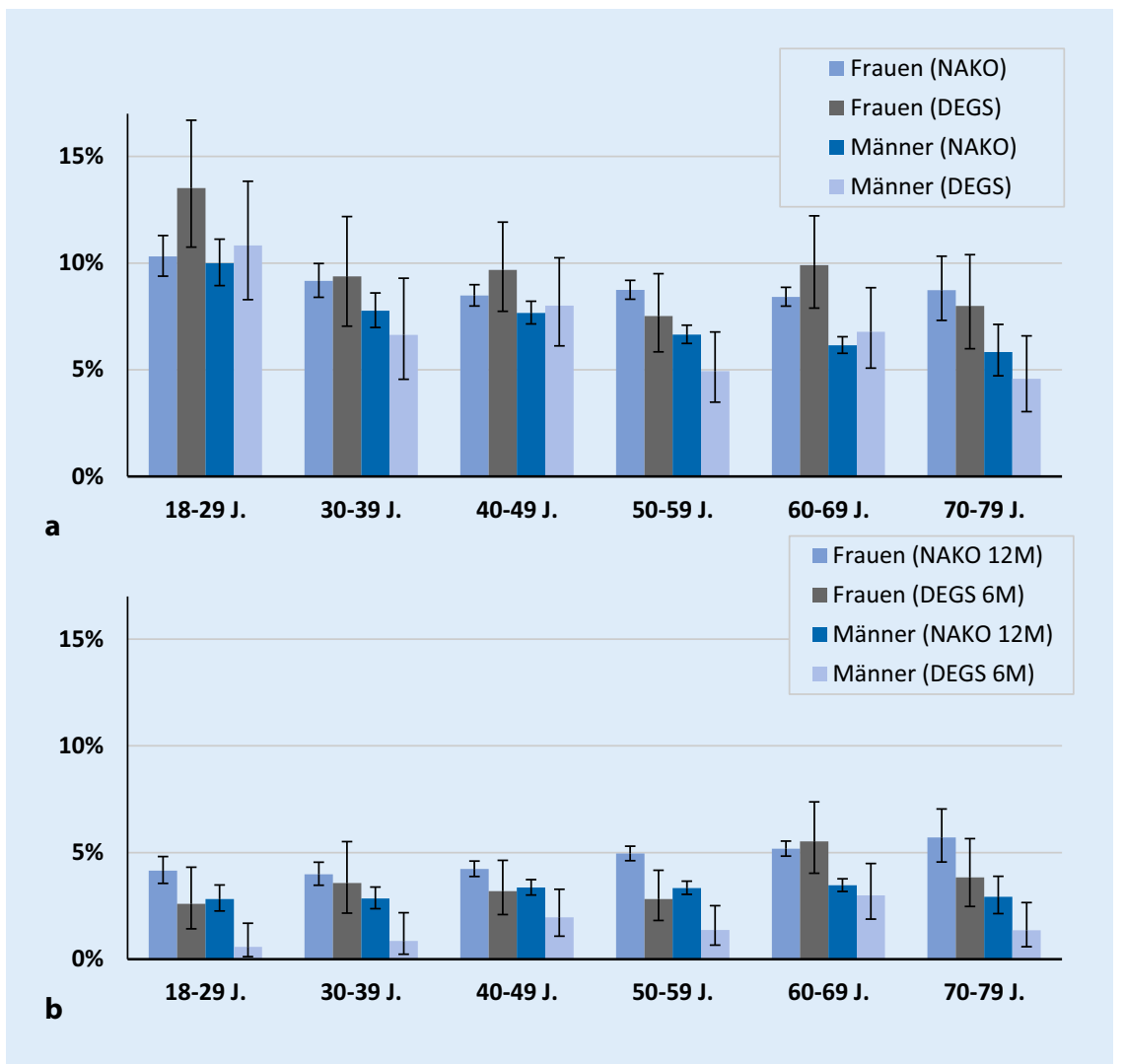

Abb. 2 A Anteil der von Asthma bronchiale betroffenen Personen in NAKO und Studie zur Gesundheit Erwachsener in Deutschland (DEGS) nach Alter und Geschlecht. a Jemals Asthma von einem Arzt diagnostiziert; $\mathbf{b}$ Asthma behandelt in den letzten 12 Monaten (NAKO) bzw. 3 Monate vor der Befragung oder in den nächsten 3 Monaten beabsichtigt (DEGS), mit jeweils $95 \%$ Konfidenzintervallen. Hinweis: Die Altersgruppe 70-79 Jahre in der NAKO umfasst Personen bis max. 75 Jahre

\section{Diskussion}

Zur Halbzeit der Basiserhebung der NAKO war die Häufigkeit der Asthmadiagnosen ähnlich wie in repräsentativen Daten für Deutschland (z.B DEGS), ebenso die Alters- und Geschlechterverteilungen. Der Anteil der aktuell wegen Asthma Behandelten lag hingegen niedriger als in den Vergleichszahlen. Zwei Drittel der Teilnehmenden mit Asthma (jemals) konnten dem AOA zugeordnet werden.

Die NAKO-Daten zeigen auch eine Übereinstimmung mit Abrechnungsdaten der Krankenkassen. In einer entsprechenden Erhebung wurde ein Anstieg der 12-Monats-Diagnoseprävalenz von knapp $5 \%$ bei 30 - bis 34 -jährigen auf $8 \%$ bei 70- bis 74 -jährigen Frauen sowie eine leicht ansteigende 12-Monats-Diagnoseprävalenz bei Männern von 4,5\% auf $5 \%$ in denselben Altersklassen verzeichnet [18]. Wenn man nur die Teil- nehmenden mit Asthma in der NAKO betrachtet, die in den letzten 12 Monaten wegen Asthma behandelt wurden, sieht man leicht reduzierte Häufigkeiten mit ähnlichem Altersverlauf.

Die retrospektiven Angaben der Teilnehmenden in der NAKO zeigen, wie auch andere internationale Quellen, dass Jungen im Kindesalter häufiger von Asthma betroffen sind als Mädchen, was sich meist nach der Pubertät umkehrt [19, 20]. Frauen sind im mittleren Alter dann fast doppelt so häufig von Asthma betroffen wie Männer gleichen Alters [5, 12]. Die Daten der NAKO sowie aus DEGS und dem Versorgungsatlas (basierend auf bundesweiten Abrechnungsdaten der vertragsärztlichen Versorgung in Deutschland; [18]) zeigen jedoch, dass Frauen im mittleren Alter nur geringfügig häufiger betroffen sind als Männer und ein deutlicherer Anstieg in der Häufigkeit von Asthma bei Frauen erst nach den Wechseljahren zu erkennen ist
[18]. Während andere Studien zeigen, dass die Asthmahäufigkeit im weiblichen Geschlecht nach den Wechseljahren wieder abnimmt [20], ist dies anhand der vorliegenden NAKO-Daten also nicht zu beobachten. Da endogene und exogene hormonelle Faktoren eine Rolle bei der Entstehung von Asthma spielen können, erscheint eine Veränderung nach den Wechseljahren plausibel [21, 22].

Trotz Hinweisen darauf, dass sich Asthma auch bei Erwachsenen entwickeln kann, die als Kind nie relevante Atemwegssymptome hatten, gibt es immer noch einige Kontroversen über die Existenz von Asthma bei Erwachsenen als eigenständiges Krankheitsbild [23]. In der vorliegenden Untersuchung gaben nur etwa ein Drittel der NAKO-Teilnehmenden an, dass die Erstdiagnose Asthma im Kindes- oder Jugendalter gestellt wurde. Bei Teilnehmenden in den ältesten Geburtsjahrgängen (1940-1949) waren es sogar nur $13,5 \%$, die eine Asthmadiagnose vor dem 18. Lebensjahr angaben. In der aktuellen Untersuchung waren $68 \%$ der Teilnehmenden mit Asthma $(N=5245)$ laut Selbstangabe von AOA betroffen. Bedeutend in diesem Zusammenhang ist, dass sich Teilnehmer mit AOA fast doppelt so häufig - in den letzten 12 Monaten einer ärztlichen Behandlung unterzogen als Teilnehmer mit COA.

Des Weiteren gaben NAKO-Teilnehmende der älteren Geburtsjahrgänge insgesamt seltener an, jemals die ärztliche Diagnose Asthma erhalten zu haben, als die jüngeren Jahrgänge. Dem können verschiedene Ursachen zugrunde liegen. Eine Ursache könnte sein, dass die Erinnerung an länger zurückliegende Diagnosen in den älteren Altersgruppen erschwert ist. Zur Erfassung von Asthma zeigte ein umfassendes Review, dass die Frage nach ärztlich diagnostiziertem Asthma eine hohe Spezifizität aufwies im Vergleich zu medizinischen Unterlagen. Hingegen lag die Sensitivität zwischen $48 \%$ und $100 \%$, mit einem Mittelwert von $68 \%[24,25]$. Es wäre naheliegend zu erwarten, dass länger zurückliegende Diagnosen, also solche in der Kindheit, insbesondere wenn inzwischen die Symptome abgemildert oder gänzlich verschwunden sind, seltener 

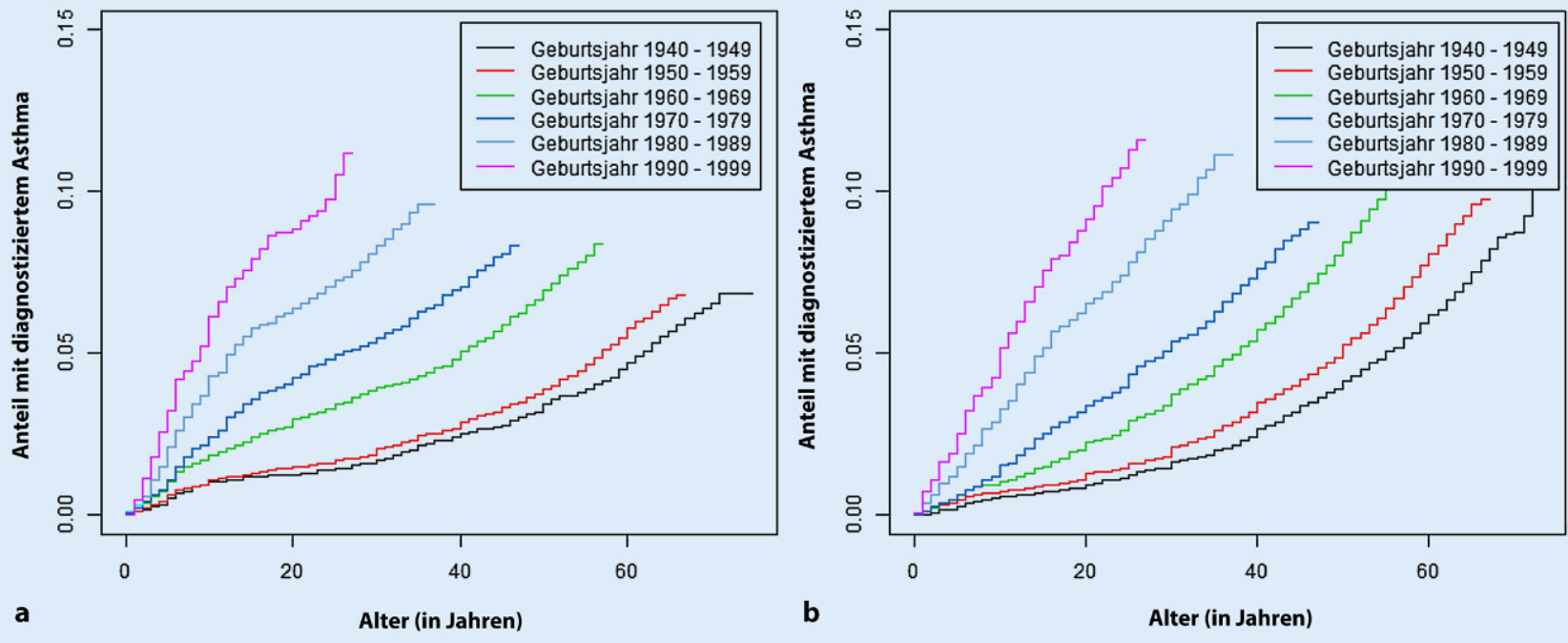

Abb. 3 A Anteil von Männern und Frauen, die bis zum Alter x die Erstdiagnose von Asthma in den verschiedenen Geburtsjahrgängen erhalten haben; a Asthma jemals diagnostiziert, Männer, b Asthma jemals diagnostiziert, Frauen

berichtet werden. Eine aktuelle Studie zeigte zwar eine hohe Reliabilität der Angabe zur Asthmadiagnose in zwei Untersuchungen im fünfährigen $\mathrm{Ab}$ stand, aber auch, dass die Reliabilität für länger zurückliegende Diagnosen in der Kindheit abnimmt [26]. Außer diesen Erklärungen in Richtung eines RecallBias sind aber auch andere Ursachen möglich, die für die niedrigeren Anteile in höheren Altersgruppen verantwortlich sein können. Es kann Periodenbzw. Kohorteneffekte bzgl. der relevanten Expositionen geben, die dazu führen, dass das tatsächliche Neuauftreten von Asthma in den älteren Altersgruppen geringer war.

In fast allen Ländern steigen die Diagnosen allergischer Erkrankungen insbesondere auch von Asthma an [27, 28]. Dabei besteht sowohl Uneinigkeit darüber, welcher Anteil lediglich auf einen Anstieg der öffentlichen Wahrnehmung beruht, als auch, was mögliche Ursachen für einen tatsächlichen Anstieg sind. Sowohl die medizinische Versorgung als auch die Diagnostik von Asthma haben sich über die Jahre hin verbessert, z. B. werden Bodyplethysmographie und Spirometrie heute deutlich häufiger als früher verwendet und damit Asthma wahrscheinlich auch häufiger diagnostiziert. Weiterhin ist eine Unterscheidung zwischen Asthma und der chronisch ob- struktiven Lungenerkrankung (COPD) insbesondere im Alter schwierig, da sich die Symptome der beiden Krankheiten sehr ähneln [7]. In Betracht ziehen sollte man auch den niedrigschwelligeren Zugang zu diagnostischen Verfahren der jüngeren Teilnehmer als eine potenzielle Erklärung des geringeren diagnostizierten Anteils von Asthma bei älteren Teilnehmern.

Ein geringerer Anteil Erkrankter in höheren Altersgruppen kann sicher auch auf eine Selektion der leichteren Fälle, die überlebt haben, zurückzuführen sein. Die Asthmatherapie hat beispielsweise in den letzten Jahrzenten einen dramatischen Wandel erfahren und damit deutliche Fortschritte gemacht [2, 29], von denen die älteren Jahrgänge aber noch nicht profitieren konnten, sodass Personen mit schwerem Asthma möglicherweise früher verstorben sind.

Weitere Selektionsmechanismen sind denkbar, z.B. dass an der Studie nur jüngere Asthmaerkrankte teilgenommen haben, weil viele ältere Menschen mit Asthma sich nicht imstande fühlten an solch einer intensiven Untersuchung teilzunehmen. Dagegen sprechen allerdings die vergleichbaren Ergebnisse des Versorgungsatlas [18], bei denen ein Selektionsbias durch die unterschiedliche Teilnahmebereitschaft weitgehend ausgeschlossen werden konnte. Insgesamt ist die end- gültige Bewertung der Ergebnisse derzeit schwierig - nicht zuletzt stehen andere Studien, die auf Fragebogenerhebungen zurückgreifen, auch vor den gleichen Fragen.

Die Unterschiede in der Häufigkeit von behandeltem Asthma in NAKO und DEGS können auch auf die hier genutzten unterschiedlichen Erfassungszeiträume zurückzuführen sein. Ebenso wären im Vergleich der Asthmahäufigkeit in NAKO und DEGS aufgrund einer unterschiedlichen Altersobergrenze, NAKO (75 Jahre) und DEGS (79 Jahre), Unterschiede möglich, aber nicht sichtbar.

In der NAKO-Stichprobe sind die Altersgruppen $>40$ Jahre gegenüber den jüngeren Altersgruppen überrepräsentiert. Da die Kernergebnisse unserer Untersuchung jedoch als altersgruppenspezifische Häufigkeiten dargestellt werden, wirkt sich diese Tatsache nur bei der altersgruppenübergreifenden Darstellung aus.

\section{Stärken und Schwächen}

Bei der NAKO handelt es sich um die größte jemals in Deutschland durchgeführte Kohortenstudie, mit ausreichenden Fallzahlen, um eine Unterscheidung von vielen Untergruppen zu ermöglichen. Einschränkend ist jedoch zu erwähnen, dass die berichteten Ergebnisse 
aus einer Teilstichprobe der NAKO stammen und Selektionseffekte innerhalb der NAKO-Stichprobe durchaus Einfluss auf die Häufigkeit der berichteten Asthmadiagnosen in dieser Auswertung haben können. Zudem können Aussagen über die Allgemeinheit bei Studien mit geringer Response zu Verzerrungen führen (Responserate bei NAKO 10-30\% je nach Studienzentrum bzw. DEGS $42 \%$ [30]).

Außerdem konnten noch keine Angaben zum sozioökonomischen Status berücksichtigt werden, der mit dem Auftreten von Asthma assoziiert ist [31]. Mit vollständigem Datensatz und unter Berücksichtigung der Charakteristika aller Personen können bessere Aussagen zur Häufigkeit von Asthma in der NAKO getroffen werden. Eine zentrale Einschränkung stellt dar, dass die Untersuchung auf Selbstangaben der erwachsenen Teilnehmenden zu einer für das Kindesalter typischen Krankheit basiert. Falsche Angaben über den Krankheitsbeginn sind bei dieser Erhebungsmethode nicht ausgeschlossen. Eine externe Validierung könnte mittels Krankenkassendaten erfolgen, müsste sich allerdings auf relativ aktuelle Ereignisse und Diagnosen beschränken. Für die Life-CourseEpidemiologie von chronischen und oft transienten Krankheiten des Kindesalters wie Asthma und Allergien sind bevölkerungsbasierte Geburtskohortenstudien besser geeignet $[32,33]$.

\section{Fazit und Ausblick}

Die vorliegenden Ergebnisse geben einen ersten Einblick in die in der NAKO erhobenen Daten zur Häufigkeit von selbstberichtetem Asthma bronchiale. In weiteren Analysen können die berichteten Diagnosen mit aktuell eingenommenen Medikamenten abgeglichen werden. Ebenso wird es möglich sein, die Daten zur Lungenfunktion sowie Ergebnisse der bildgebenden Verfahren (MRT) zur Bewertung der Erkrankungsschwere hinzuzuziehen und den weiteren Verlauf der bereits diagnostizierten Erkrankungen und Risikofaktoren für neu auftretendes Asthma zu analysieren.

\section{Korrespondenzadresse}

\section{Dr. rer. nat. Cornelia Gottschick}

Institut für Medizinische Epidemiologie, Biometrie und Informatik, Martin-LutherUniversität Halle-Wittenberg Magdeburger Str. 8, 06112 Halle (Saale), Deutschland

cornelia.gottschick@uk-halle.de

Danksagung. Wir danken allen Teilnehmerinnen und Teilnehmern sowie den Mitarbeiterinnen und Mitarbeitern der NAKO Gesundheitsstudie.

Förderung. Dieses Projekt wurde mit Daten der NAKO Gesundheitsstudie durchgeführt (www.nako. de). Die NAKO Gesundheitsstudie wird durch das Bundesministerium für Bildung und Forschung (BMBF, Förderkennzeichen 01ER1301A/B/C und 01ER1511D), die Bundesländer und die HelmholtzGemeinschaft gefördert sowie durch die beteiligten Universitäten und Institute der Leibniz-Gemeinschaft finanziell unterstützt.

Funding. Open Access funding provided by Projekt DEAL.

\section{Einhaltung ethischer Richtlinien}

Interessenkonflikt. S. Langer, J. Horn, A. Kluttig, R. Mikolajczyk, S. Karrasch, H. Schulz, H.-E. Wichmann, J. Linseisen, L. Jaeschke, T. Pischon, J. Fricke, T. Keil, W. Ahrens, K. Günther, O. Kuß, T. Schikowski, B.Schmidt, K.-H. Jöckel, K.B. Michels, C.-W. Franzke, H. Becher, A. Jagodzinski, S. Castell, Y. Kemmling, W. Lieb, S. Waniek, K. Wirkner, M. Löffler, R. Kaaks, K.H. Greiser, K. Berger, N. Legath, C. Meinke-Franze, S. Schipf, M. Leitzmann, H. Baurecht, K. Weigl, E. Amitay und C. Gottschick geben an, dass kein Interessenkonflikt besteht.

Alle beschriebenen Untersuchungen am Menschen oder an menschlichem Gewebe wurden mit Zustimmung der zuständigen Ethikkommission, im Einklang mit nationalem Recht sowie gemäß der Deklaration von Helsinki von 1975 (in der aktuellen, überarbeiteten Fassung) durchgeführt. Von allen beteiligten Teilnehmenden liegt eine Einverständniserklärung vor.

Open Access. Dieser Artikel wird unter der Creative Commons Namensnennung 4.0 International Lizenz veröffentlicht, welche die Nutzung, Vervielfältigung, Bearbeitung, Verbreitung und Wiedergabe in jeglichem Medium und Format erlaubt, sofern Sie den/die ursprünglichen Autor(en) und die Quelle ordnungsgemäßnennen, einen Link zur Creative Commons Lizenz beifügen und angeben, ob Änderungen vorgenommen wurden.

Die in diesem Artikel enthaltenen Bilder und sonstiges Drittmaterial unterliegen ebenfalls der genannten Creative Commons Lizenz, sofern sich aus der Abbildungslegende nichts anderes ergibt. Sofern das betreffende Material nicht unter der genannten Creative Commons Lizenz steht und die betreffende Handlung nicht nach gesetzlichen Vorschriften erlaubt ist, ist für die oben aufgeführten Weiterverwendungen des Materials die Einwilligung des jeweiligen Rechteinhabers einzuholen.
Weitere Details zur Lizenz entnehmen Sie bitte der Lizenzinformation auf http://creativecommons.org/ licenses/by/4.0/deed.de.

\section{Literatur}

1. Steppuhn H, Kuhnert R, Scheidt-Nave C (2017) 12-Monats-Prävalenz von Asthma bronchiale bei Erwachsenen in Deutschland. Epidemiologie und Gesundheitsberichterstattung. Robert KochInstitut, Berlin

2. Virchow JC (2010) Asthma - Geschichtliche Entwicklung, Status quo und Ausblick. Pneumologie 64:541-549

3. Nowak D, Von Mutius E (2004) Bronchial asthma in children and adults: risk factors, diagnosis and standard treatment. Dtsch Med Wochenschr 129:509-516

4. Masoli M, Fabian D, Holt S, Beasley R (2004) The global burden of asthma: executive summary of the GINA Dissemination Committee report. Allergy 59:469-478

5. Bisgaard H, Bonnelykke K (2010) Long-term studies of the natural history of asthma in childhood. J Allergy Clin Immunol 126:187-197

6. Ayres JG (1990) Late onset asthma. BMJ 300:1602-1603

7. Hirano T, Matsunaga K (2018) Late-onset asthma: current perspectives. J Asthma Allergy 11:19-27

8. Dunn RM, Busse PJ, Wechsler ME (2018) Asthma in the elderly and late-onset adult asthma. Allergy 73:284-294

9. Gibson PG, Mcdonald VM, Marks GB (2010) Asthma in older adults. Lancet 376:803-813

10. De Nijs SB, Venekamp LN, Bel EH (2013) Adultonset asthma: is it really different? Eur Respir Rev 22:44-52

11. Amelink M, De Nijs SB, Berger M et al (2012) Nonatopic males with adult onset asthma are at risk of persistent airflow limitation. Clin Exp Allergy 42:769-774

12. Leynaert B, Sunyer J, Garcia-Esteban R et al (2012) Gender differences in prevalence, diagnosis and incidence of allergic and non-allergic asthma: a population-based cohort. Thorax 67:625-631

13. Bauer BA, Reed CE, Yunginger JW, Wollan PC, Silverstein MD (1997) Incidence and outcomes of asthma in the elderly. A population-based study in Rochester, Minnesota. Chest 111:303-310

14. Langen U, SchmitzR, Steppuhn H (2013) Häufigkeit allergischer Erkrankungen in Deutschland. Epidemiologie und Gesundheitsberichterstattung. Robert Koch-Institut, Berlin

15. Wichmann H-E, Kaaks R, Hoffmann W, Jöckel K-H, Greiser KH, Linseisen J (2012) Die Nationale Kohorte. Bundesgesundheitsblatt Gesundheitsforschung Gesundheitsschutz 55:781-789

16. German National Cohort Consortium (2014) The German National Cohort: aims, study design and organization. Eur JEpidemiol 29:371-382

17. Robert Koch-Institut (2015) Studie zur Gesundheit Erwachsener in Deutschland (DEGS1). Robert Koch-Institut, Berlin

18. Akmatov M, Holstiege J, Steffen A, Bätzing J (2018) Diagnoseprävalenz und -inzidenz von Asthma bronchiale - Ergebnisse einer Studie mit Versorgungsdaten aller gesetzlich Versicherten in Deutschland (2009-2016). In: Versorgungsatlas. Zentralinstitut für die kassenärztliche Versorgung in Deutschland (Zi), Berlin (https://www.versorgungsatlas.de/themen/alle- 
analysen-nach-datum-sortiert/?tab=6\&uid=92. Zugriffam 11.11.2019)

19. Fitzpatrick AM, Teague WG, Meyers DA et al (2011) Heterogeneity of severe asthma in childhood: confirmation by cluster analysis of children in the National Institutes of Health/ National Heart, Lung, and Blood Institute Severe Asthma Research Program. J Allergy Clin Immuno 127(313):382-389.e1-13

20. Carey MA, Card JW, VoltzJW etal (2007) It's all about sex: gender, lung development and lung disease. Trends Endocrinol Metab 18:308-313

21. Bennett WD, Ivins S, Alexis NE et al (2016) Effect of obesity on acute ozone-induced changes in airway function, reactivity, and inflammation in adult females. PLoSONE 11:e160030

22. Fuseini $H$, Newcomb DC (2017) Mechanisms driving gender differences in asthma. Curr Allergy Asthma Rep 17:19

23. Bush A, Menzies-Gow A (2009) Phenotypic differences between pediatric and adult asthma. Proc Am Thorac Soc 6:712-719

24. Toren K, Brisman J, Jarvholm B (1993) Asthma and asthma-like symptoms in adults assessed by questionnaires. A literature review. Chest 104:600-608

25. Toren K, Palmqvist $M$, Lowhagen O, Balder B, Tunsater A (2006) Self-reported asthma was biased in relation to disease severity while reported year of asthma onset was accurate. J Clin Epidemiol 59:90-93

26. Mirabelli MC, Beavers SF, Flanders WD, Chatterjee $A B$ (2014) Reliability in reporting asthma history and age at asthma onset. J Asthma 51:956-963

27. Loftus PA, Wise SK (2016) Epidemiology of asthma. Curr Opin Otolaryngol Head NeckSurg 24:245-249

28. Lundback B, Backman H, Lotvall J, Ronmark E (2016) Is asthma prevalence still increasing? Expert Rev Respir Med 10:39-51

29. Hasford J, Virchow JC (2006) Excess mortality in patients with asthma on long-acting beta2agonists. Eur Respir J 28:900-902

30. Kamtsiuris P, Lange M, Hoffmann R et al (2013) Die erste Welle der Studiezur GesundheitErwachsener in Deutschland (DEGS1). Epidemiologie und Gesundheitsberichterstattung. Robert KochInstitut, Berlin

31. Trivedi M, Denton E (2019) Asthma in children and adults-what are the differences and what can they tell us about asthma? Front Pediatr 7:256

32. Bousquet J, Gern JE, Martinez FD et al (2014) Birth cohorts in asthma and allergic diseases: report of a NIAID/NHLBI/MeDALL joint workshop. J Allergy Clin Immunol 133:1535-1546

33. Bousquet J, Anto J, Sunyer J, Nieuwenhuijsen M, Vrijheid M, Keil T (2013) Pooling birth cohorts in allergy and asthma: European Union-funded initiatives-a MeDALL, CHICOS, ENRIECO, and GA(2)LEN joint paper. Int Arch Allergy Immunol 161:1-10

\section{Affiliations}

${ }^{1}$ Institut für Medizinische Epidemiologie, Biometrie und Informatik, Martin-Luther-Universität HalleWittenberg, Halle (Saale), Deutschland; ' ${ }^{2}$ Institut für Epidemiologie, Helmholtz Zentrum München, Deutsches Forschungszentrum für Gesundheit und Umwelt, München, Deutschland; ${ }^{3}$ Institut und Poliklinik für Arbeits-, Sozial- und Umweltmedizin, Klinikum der Universität München, München, Deutschland; ${ }^{4}$ Comprehensive Pneumology Center Munich (CPC-M), Mitglied des Deutschen Zentrums für Lungenforschung (DZL), München, Deutschland; ${ }^{5}$ Ludwig-Maximilians-Universität München, Lehrstuhl für Epidemiologie, UNIKA-T Augsburg, Augsburg, Deutschland; ${ }^{6}$ Klinische Epidemiologie, Helmholtz Zentrum München, Deutsches Forschungszentrum für Gesundheit und Umwelt, München, Deutschland; ' ${ }^{7}$ Forschergruppe Molekulare Epidemiologie, Max-Delbrück-Centrum für Molekulare Medizin in der Helmholtz-Gemeinschaft (MDC), Berlin, Deutschland; ${ }^{8}$ Charité - Universitätsmedizin Berlin, Berlin, Deutschland; ' Deutsches Zentrum für Herz-Kreislauf-Forschung (DZHK), Partnerstandort Berlin, Berlin, Deutschland; ${ }^{10} \mathrm{MDC} / \mathrm{BIH}$ Biobank, Max-Delbrück-Centrum für Molekulare Medizin in der Helmholtz-Gemeinschaft (MDC) und Berlin Institute of Health (BIH), Berlin, Deutschland; " Institut für Sozialmedizin, Epidemiologie und Gesundheitsökonomie, Charité Universitätsmedizin Berlin, Berlin, Deutschland; ${ }^{12}$ Institut für Klinische Epidemiologie und Biometrie, Universität Würzburg, Würzburg, Deutschland; ${ }^{13}$ Landesinstitut für Gesundheit, Bayerisches Landesamt für Gesundheit und Lebensmittelsicherheit, Bad Kissingen, Deutschland; ${ }^{14}$ Leibniz-Institut für Präventionsforschung und Epidemiologie - BIPS, Bremen, Deutschland; ${ }^{15}$ Institut für Statistik, Fachbereich Mathematik und Informatik, Universität Bremen, Bremen, Deutschland; ${ }^{16}$ Leibniz-Zentrum für Diabetes-Forschung an der Heinrich-Heine-Universität Düsseldorf, Institut für Biometrie und Epidemiologie, Deutsches DiabetesZentrum (DDZ), Düsseldorf, Deutschland; ${ }^{17}$ IUF - Leibniz-Institut für umweltmedizinische Forschung gGmbH, Düsseldorf, Deutschland; ${ }^{18}$ Institut für Medizinische Informatik, Biometrie und Epidemiologie, Universitätsklinikum Essen, Essen, Deutschland; ${ }^{19}$ Institut für Prävention und Tumorepidemiologie, Universitätsklinikum Freiburg, Medizinische Fakultät, Albert-Ludwigs-Universität Freiburg, Freiburg, Deutschland; ${ }^{20}$ Institut für Medizinische Biometrie und Epidemiologie, Universitätsklinikum HamburgEppendorf, Hamburg, Deutschland; ${ }^{21}$ Klinik für Allgemeine und Interventionelle Kardiologie, Universitäres Herz- und Gefäßzentrum Hamburg - Eppendorf, Hamburg, Deutschland; ${ }^{22}$ Deutsches Zentrum für Herzkreislaufforschung, Hamburg, Deutschland; ${ }^{23}$ Epidemiologisches Studienzentrum, Universitätsklinikum Hamburg - Eppendorf, Hamburg, Deutschland; ${ }^{24} \mathrm{Helmholtz-Zentrum} \mathrm{für}$ Infektionsforschung, Braunschweig, Deutschland; ${ }^{25}$ Institut für Epidemiologie, Christian-AlbrechtsUniversität Kiel, Kiel, Deutschland; ${ }^{26}$ Institut für Medizinische Informatik, Statistik und Epidemiologie (IMISE), Universität Leipzig, Leipzig, Deutschland; ${ }^{27}$ LIFE-Forschungszentrum für Zivilisationskrankheiten, Universität Leipzig, Leipzig, Deutschland; ${ }^{28}$ Abteilung Epidemiologie von Krebserkrankungen, Deutsches Krebsforschungszentrum (DKFZ), Heidelberg, Deutschland; ${ }^{29}$ Institut für Epidemiologie und Sozialmedizin, Westfälische Wilhelms-Universität Münster, Münster, Deutschland; ${ }^{30}$ Institut für Community Medicine, Universitätsmedizin Greifswald, Greifswald, Deutschland; ${ }^{31}$ Institut für Epidemiologie und Präventivmedizin, Universität Regensburg, Regensburg, Deutschland; ${ }^{32} \mathrm{Abt}$. Klinische Epidemiologie und Alternsforschung, Deutsches Krebsforschungszentrum (DKFZ), Heidelberg, Deutschland 